\title{
Aspirin inhibits glucose-6-phosphate dehydrogenase activity in HCT 116 cells through acetylation: Identification of aspirin-acetylated sites
}

\author{
GUOQIANG AI $^{1}$, RAKESH DACHINENI ${ }^{1}$, D. RAMESH KUMAR ${ }^{1,2}$, \\ LLOYD F. ALFONSO $^{3}$, SRINIVASAN MARIMUTHU ${ }^{1,4}$ and G. JAYARAMA BHAT ${ }^{1}$ \\ ${ }^{1}$ Department of Pharmaceutical Sciences, South Dakota State University College of Pharmacy, \\ Avera Health and Sciences Center, Brookings, SD 57007; ${ }^{3}$ D'Youville College School of Pharmacy, Buffalo, NY 14201, USA
}

Received February 19, 2016; Accepted May 18, 2016

DOI: $10.3892 / \mathrm{mmr} .2016 .5449$

\begin{abstract}
Glucose-6-phosphate dehydrogenase (G6PD) catalyzes the first reaction in the pentose phosphate pathway, and generates ribose sugars, which are required for nucleic acid synthesis, and nicotinamide adenine dinucleotide phosphate (NADPH), which is important for neutralization of oxidative stress. The expression of G6PD is elevated in several types of tumor, including colon, breast and lung cancer, and has been implicated in cancer cell growth. Our previous study demonstrated that exposure of HCT 116 human colorectal cancer cells to aspirin caused acetylation of G6PD, and this was associated with a decrease in its enzyme activity. In the present study, this observation was expanded to HT-29 colorectal cancer cells, in order to compare aspirin-mediated acetylation of G6PD and its activity between HCT 116 and HT-29 cells. In addition, the present study aimed to determine the acetylation targets of aspirin on recombinant G6PD to provide an insight into the mechanisms of inhibition. The results demonstrated that the extent of G6PD acetylation was significantly higher in HCT 116 cells compared with in HT-29 cells; accordingly, a greater reduction in G6PD enzyme activity was observed in the HCT 116 cells. Mass spectrometry analysis of aspirin-acetylated G6PD (isoform $a$ ) revealed that aspirin acetylated a total of 14 lysine residues, which were dispersed throughout the length of the G6PD protein. One of the important amino acid targets of aspirin
\end{abstract}

Correspondence to: Associate Professor G. Jayarama Bhat, Department of Pharmaceutical Sciences, South Dakota State University College of Pharmacy, Avera Health and Sciences Center, 1055 Campanile Avenue, Brookings, SD 57007, USA

E-mail: jayarama.gunaje@sdstate.edu

Present addresses: ${ }^{2}$ Aquatic Animal Health Division, Central Institute of Brackishwater Aquaculture, Chennai, Tamil Nadu 600028, India; ${ }^{4}$ Ayurveda Research Institute for Mother \& Child Health Care, Poojapura, Trivandrum, Kerala 695012, India

Key words: chemoprevention, colorectal cancer, pentose phosphate pathway, glucose-6-phosphate dehydrogenase, aspirin, acetylation included lysine 235 (K235, in isoform $a$ ) and this corresponds to K205 in isoform $b$, which has previously been identified as being important for catalysis. Acetylation of G6PD at several sites, including K235 (K205 in isoform b), may mediate inhibition of G6PD activity, which may contribute to the ability of aspirin to exert anticancer effects through decreased synthesis of ribose sugars and NADPH.

\section{Introduction}

It is well known from epidemiological studies that regular intake of aspirin reduces the risk of cancer of the epithelial tissues, with the most profound protective effect being observed against colon cancer (1-5). Evidence that aspirin decreases the occurrence of epithelial cancer is compelling; however, the pathways and proteins involved in this process remain to be elucidated, and numerous mechanisms and targets have been proposed (5-7). Aspirin consists of two functional groups: The acetyl group and the salicylate group, both of which have been implicated in its anticancer effects. Early investigations in this area have predominantly focused on the ability of aspirin to acetylate cyclooxygenases (COX), leading to inactivation of enzyme activity, as a primary mechanism to explain its anticancer effects (2). This is because inactivation of COX induces decreased prostaglandin synthesis and inflammation, which has been linked to decreased cancer occurrence. However, other studies have reported that several COX-independent mechanisms involving salicylic acid, the primary metabolite of aspirin, may also contribute to its anticancer effects. Some of the direct binding targets of salicylic acid that have been identified to date include: I $\kappa$ B kinase $\beta(8)$, AMP-activated protein kinase (9), high mobility group box 1 proteins (10) and cyclin dependent kinase 2 (11). It is argued that modulation of the functional activity of these proteins by salicylic acid may contribute to the anticancer effects of aspirin.

Previous studies from our laboratory and others have demonstrated that exposure of cancer cells to aspirin induces acetylation of hundreds of proteins (12-15). The acetylation targets of aspirin identified in our previous studies included the tumor suppressor protein p53 (16,17), enzymes of the glycolytic pathway, cytoskeletal proteins, histones, and ribosomal and 
mitochondrial proteins (13). The enzymes that were identified in the glycolytic pathway include aldolase, glyceraldehyde-3-phosphate dehydrogenase, enolase, pyruvate kinase M2 and lactate dehydrogenase $\mathrm{A}$ and $\mathrm{B}$ chains. In addition, we revealed that aspirin acetylated glucose-6-phosphate dehydrogenase (G6PD) and transketolase enzymes in the pentose phosphate pathway (13). Assays carried out for some of the acetylated glycolytic pathway enzymes indicated that acetylation in the majority of cases does not affect enzyme activity, thus suggesting that it probably has a neutral impact on bulk protein functions. However, acetylation of G6PD was associated with a decrease in its enzyme activity, thus suggesting that aspirin potentially modulates G6PD activity.

G6PD is an important regulatory enzyme in the pentose phosphate pathway that produces ribose-5-phosphate, which is essential for nucleic acid synthesis in rapidly dividing cells $(18,19)$. G6PD also produces the reducing agent nicotinamide adenine dinucleotide phosphate (NADPH), which is essential for the neutralization of oxygen free radicals and the reductive biosynthesis of fatty acids. Therefore, G6PD has an important role under normal physiological conditions, as well as in cancer cell growth. Within human cells, two isoforms of G6PD have been reported (20). G6PD isoform $a$ has a total of 545 amino acids, whereas isoform $b$ has a total of 515 amino acids. Isoform $b$ is identical to isoform $a$ except that it lacks the first 30 amino acids in the NH2 terminus. It has been reported that isoform $b$ represents the functionally active G6PD enzyme (20) and accordingly, the majority of previous studies has reported on this isoform (21). Our earlier observation that aspirin acetylated G6PD in HCT 116 colorectal cancer cells, leading to a decrease in its enzyme activity, suggested the possibility that it may have a role in the chemopreventive actions of aspirin (13). The present study extended these earlier observations to HT-29 human colorectal cancer cells and compared aspirin-mediated acetylation of G6PD and enzyme activity between HCT 116 and HT-29 cells. The present study demonstrated that compared with HCT 116 cells, HT-29 cells contained significantly lower levels of acetylated G6PD; however, in both cell types, G6PD protein expression levels were similar. To gain insight into how aspirin decreases G6PD activity through acetylation, mass spectrometry (MS) analysis was used to identify the aspirin-acetylated sites on recombinant G6PD. The results demonstrated that exposure of recombinant G6PD to aspirin induced acetylation of 14 lysine residues. These lysine targets were found to be localized throughout the G6PD protein, and included the NAD-binding domain as well as the C-terminal domain. The important targets of aspirin included lysine 235 (K235), which is present in the highly conserved peptide RIDHYLGK (aa 228-235 in isoform $a$ ) in G6PD. K235 in isoform $a$ corresponds to K205 in isoform $b$, which has previously been demonstrated to be essential for catalysis (21). It is likely that inhibition of G6PD enzyme activity by aspirin is due to acetylation of this key lysine residue (K235 in isoform $a$ and $\mathrm{K} 205$ in isoform $b$ ). Lysine acetylation at K205 (or K235) may prevent substrate binding (glucose-6-phosphate) into the active site, rendering the enzyme catalytically inactive. Alternatively, acetylation of other lysines in the nucleotide-binding domain may also lead to decreased enzyme activity in cells treated with aspirin.

\section{Materials and methods}

Materials. Cell culture reagents were purchased from Invitrogen (Thermo Fisher Scientific, Inc., Waltham, MA, USA). Aspirin was obtained from Sigma-Aldrich (St. Louis, MO, USA). Anti-acetyl lysine antibody (cat. no. 9441S; 1:5,000 dilution) was purchased from Cell Signaling Technology, Inc. (Danvers, MA, USA), and anti-G6PD antibody (cat. no. sc-46971; 1:2:000 dilution) was purchased from Santa Cruz Biotechnology, Inc. (Dallas, TX, USA). The recombinant G6PD protein (isoform $a$ ) was obtained from Origene Technologies, Inc. (Rockville, MD, USA). The G6PD activity colorimetric assay kit was obtained from Biovision, Inc. (Milpitas, CA, USA). All other chemicals were purchased from either Sigma-Aldrich or Thermo Fisher Scientific, Inc.

Cell culture. HCT 116 and HT-29 colorectal cancer cells were obtained from American Type Culture Collection (Manassas, VA, USA). The cells were routinely cultured in McCoy's 5A medium (Thermo Fisher Scientific, Inc.) supplemented with $10 \%$ fetal bovine serum (Thermo Fisher Scientific, Inc.) at $37^{\circ} \mathrm{C}$ with $5 \% \mathrm{CO}_{2}$ atmosphere. Cells were cultured for $12-16 \mathrm{~h}$ prior to the addition of aspirin $(0.25-2.5 \mathrm{mM})$ for the indicated times.

Preparation of cell lysates, immunoprecipitation and western blotting. HCT 116 and HT-29 cells were grown for 12-16 h at $50 \%$ confluence, and treated with aspirin for the indicated times. Cells were washed with phosphate-buffered saline and scraped in lysis buffer, then protein concentrations were estimated by Bradford protein assay, as previously described (13). A total of $200 \mu \mathrm{g}$ total protein was immunoprecipitated with agarose-conjugated anti-acetyl lysine antibody overnight at $4^{\circ} \mathrm{C}$, and was then washed three times with lysis buffer. The agarose-bound proteins were eluted using trimethylamine buffer and were immunoblotted with the anti-G6PD antibody as previously described (13), and were immunoblotted with the anti-G6PD antibody. Alternatively, $50 \mu \mathrm{g}$ total proteins were loaded onto an $8 \%$ SDS-polyacrylamide gel, and were immunoblotted the anti-G6PD antibody. Subsequently, immunoreactive bands were detected using a chemiluminescence system (Thermo Fisher Scientific, Inc.).

G6PD assay. Subconfluent cells were left untreated or were treated with the indicated concentrations of aspirin. The cells were then lysed using the assay buffer. A total of $100 \mu \mathrm{g}$ of sample from each treatment condition was used for the G6PD assay, which was performed in a 96-well plate according to the manufacturer's protocol (Biovision, Inc.). Following termination of the reaction, absorbance was measured at $450 \mathrm{~nm}$.

Sample preparation for MS analysis, using liquid chromatography-MS/MS. The recombinant G6PD isoform a $(5 \mu \mathrm{g}$; long form, NP_000393) was acetylated by incubating with $0.25 \mathrm{mM}$ aspirin for $12 \mathrm{~h}$ at room temperature, in the presence of $50 \mathrm{mM}$ MOPS ( $\mathrm{pH} 7.4$ ), in a final volume of $33 \mu 1$. For the negative control, an equal amount of protein was left untreated under the same conditions. An aliquot of acetylated protein was analyzed by immunoblotting with anti-acetyl lysine antibody. The remaining acetylated G6PD was subjected to MS as previous described (17). 
A

HCT 116

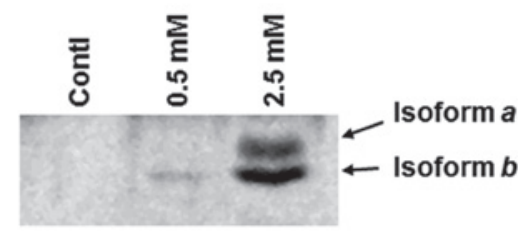

B

HT-29

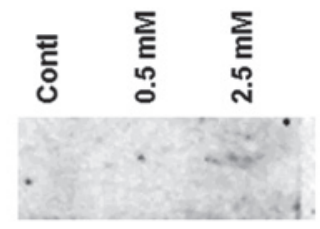

\section{IP: anti-acetyl lysine antibody \\ Probe: G6PD antibody}

C

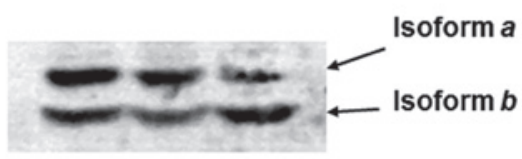

D

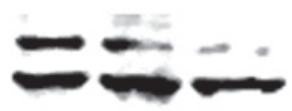

\section{Probe: G6PD antibody}

Figure 1. Aspirin-mediated acetylation of glucose-6-phosphate dehydrogenase (G6PD) is greater in (A) HCT 116 cells compared with in (B) HT-29 cells. Subconfluent cells were left untreated or were treated with aspirin for $24 \mathrm{~h}$, lysates were prepared, and equal amounts of protein were immunoprecipitated with rabbit agarose-conjugated anti-acetyl lysine antibody. Agarose-bound proteins were eluted and immunoblotted with anti-G6PD antibody. (C) HCT-116 and (D) HT-29 samples were immunoblotted with anti-G6PD antibody. The experiments were repeated three times.

Protein modeling. The FASTA sequence of recombinant G6PD (NP_000393) was obtained from the National Center of Biotechnology Information database. This sequence was then submitted to the SAM protein modeling server (22). The coordinates of the atoms obtained via email from the server were transcribed and saved as a text file. The text file was then opened in Rasmol protein modeling software (version 2.7.5). The locations of lysine were mapped using command line in the software.

Statistical analysis. All experiments were repeated 3-6 times independently. One-way analysis of variance followed by Newman-Keuls multiple comparison tests were performed to compare group differences to the control using Minitab software (version 16.0; Minitab, Inc., State College, PA, USA). P $<0.05$ was considered to indicate a statistically significant difference.

\section{Results}

Correlation between G6PD acetylation status and activity in HCT 116 and HT-29 cells. To compare the levels of aspirin-mediated G6PD acetylation between HCT 116 and HT-29 cells, the cells were left untreated or were treated with two different concentrations of aspirin $(0.5$ and $2.5 \mathrm{mM})$ for $24 \mathrm{~h}$. The lysates were then immunoprecipitated with anti-acetyl lysine antibody, and immunoblotted with anti-G6PD antibody. As shown in Fig. 1A, aspirin-induced acetylation of G6PD was detected at $0.5 \mathrm{mM}$ in HCT 116 cells; however, markedly increased levels were detected at $2.5 \mathrm{mM}$. Conversely, in the HT-29 cells, G6PD acetylation was not observed at all at $0.5 \mathrm{mM}$, and was barely detected at $2.5 \mathrm{mM}$ (Fig. 1B). As presented in Fig. 1C and D both isoforms of G6PD (isoforms $a$ and $b$ ) were detected in HCT 116 and HT-29 cells. G6PD activity was then detected in lysates prepared from both HCT 116 and HT-29 cells. Aspirin progressively inhibited G6PD activity in HCT 116 cells beginning at $0.5 \mathrm{mM}$; however, inhibition was not so strong in HT-29

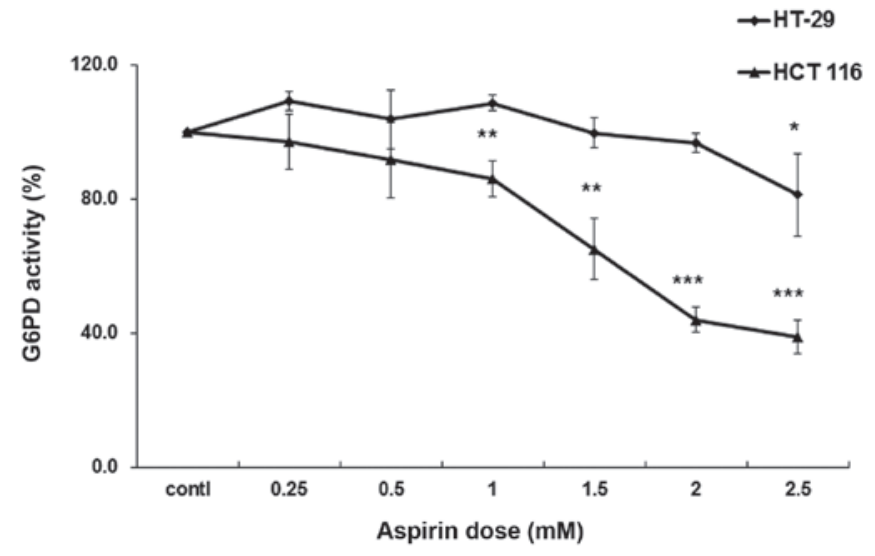

Figure 2. Effects of aspirin on glucose-6-phosphate dehydrogenase (G6PD) activity in HCT 116 and HT-29 cells. Cells were cultured and left untreated or were treated with aspirin for $24 \mathrm{~h}$. Protein $(100 \mu \mathrm{g})$ was used to conduct a G6PD assay. The reaction mixture was incubated at $37^{\circ} \mathrm{C}$ for $30 \mathrm{~min}$, and absorbance was measured at $450 \mathrm{~nm}$. G6PD activity was expressed as a percentage of control. The experiments were repeated three times. Data are represented as mean \pm standard deviation. ${ }^{*} \mathrm{P}<0.05,{ }^{* *} \mathrm{P}<0.01,{ }^{* * *} \mathrm{P}<0.001$ vs. the control.

cells (Fig. 2). These results suggest that aspirin-mediated acetylation may lead to inhibition of G6PD activity within the cellular milieu.

In vitro acetylation of recombinant G6PD protein by aspirin. The human G6PD enzyme (isoform $a$, long form) has a total of 29 lysine residue in its 545 amino acid long sequence. To determine which of the lysines are targeted by aspirin, the commercially obtained purified recombinant G6PD was acetylated with aspirin in vitro, and the product was subjected to MS analysis. Briefly, $5 \mu \mathrm{g}$ purified G6PD protein was incubated with $0.25 \mathrm{mM}$ aspirin for $12 \mathrm{~h}$; an aliquot $(5 \mathrm{ng}$ ) of the sample was initially analyzed by immunoblotting with anti-acetyl 
A

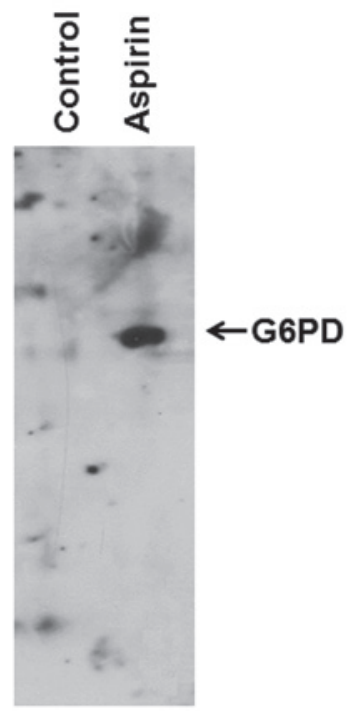

Probe: anti-acetyl lysine antibody

C

\section{K201}

IIVEK*PFGR

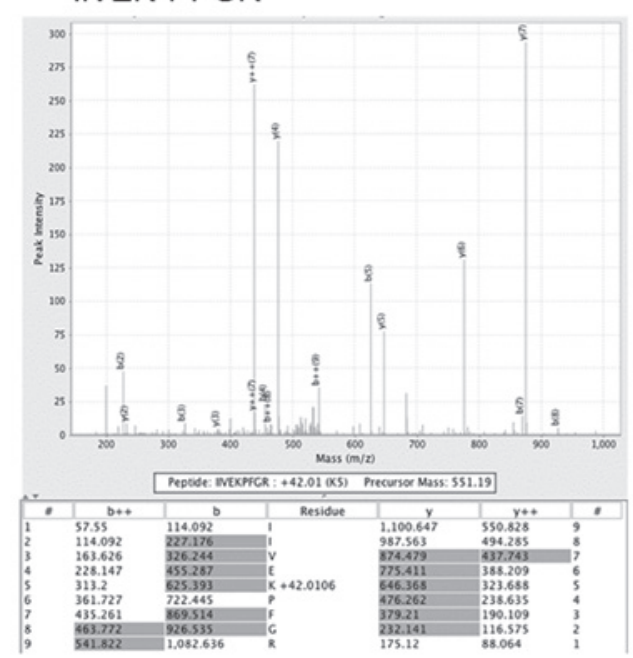

B

K77

K*IYPTIWWLFR : +42.01 (K1)

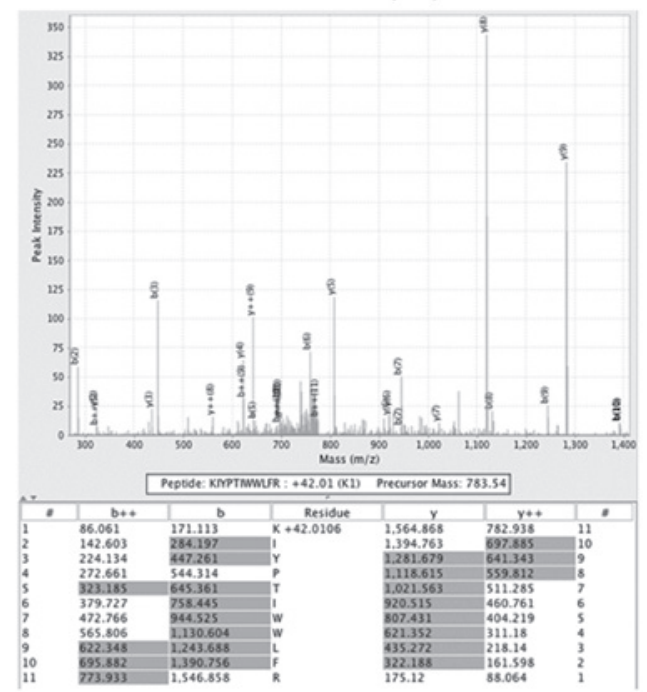

D

\section{K235}

IDHYLGK*

IDHYLGKEMVQNLMVLR 17

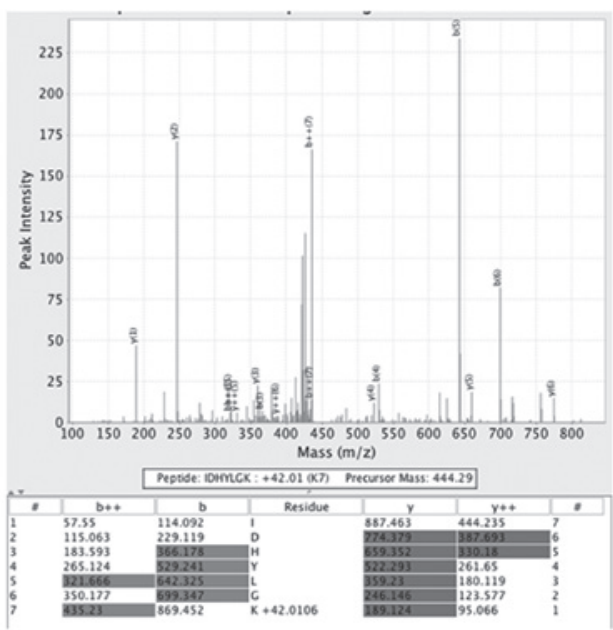

Figure 3. Mass spectrometry (MS) analysis of recombinant glucose-6-phosphate dehydrogenase (G6PD) isoform $a$. In vitro acetylation of recombinant G6PD by aspirin. (A) A total of $5 \mathrm{ng}$ in vitro acetylated recombinant G6PD was immunoblotted with anti-acetyl lysine antibody and the protein band was detected by enhanced chemiluminescence. (B-D) MS/MS fragmentation spectra showing acetyl modification of (B) K77, (C) K201 and (D) K235.

lysine antibody to ensure acetylation. As shown in Fig. 3A, anti-acetyl antibodies prominently detected a $56 \mathrm{kDa}$ protein in the aspirin-treated conditions, which was not detected in the untreated control group.

Identification of aspirin-acetylated sites on recombinant $G 6 P D$. An aliquot of the in vitro acetylated G6PD (isoform $a$ ), as presented in the blot in Fig. 3A, was subjected to MS analysis for the identification of acetylation sites. Based on the MS analysis, a total of 14 lysine residues (K77, K112, K119, K201, K235, K390, K396, K416, K438, K459, K462, K527, K538, K544) were identified as targets of aspirin-mediated acetylation. The spectra obtained for three of the acetylated lysine-containing peptides are shown in Fig. 3B-D. The spectra for other acetylated peptides are not shown. The acetylated peptides following digestion with trypsin and chymotrypsin are shown in Table I. The location of aspirin-acetylated lysine residues identified in the present study, and the comparison to naturally acetylated lysines on G6PD are shown in Table II. The peptide digests covered $86 \%$ of the total G6PD sequence. Notably, the lysine at position 235 (K235) (corresponding to K205 in G6PD isoform b), which is located in the active site of the enzyme within the evolutionarily conserved peptide RIDHYLGK (aa 228-235), is acetylated by aspirin. K205 (in isoform $b$ ) has previously been demonstrated to be important for catalysis (21). It is likely that acetylation of this residue is responsible for the observed decrease in G6PD activity in HCT 116 cells.

Molecular modeling of recombinant G6PD (NCBI accession no: NP_000393) demonstrated that the majority of the aspirin-acetylated lysine residues are surface-exposed/solvent 
Table I. Aspirin-acetylated peptides identified from glucose-6-phosphate dehydrogenase after trypsin and chymotrypsin digestion.

\begin{tabular}{lrll}
$\begin{array}{l}\text { Acetylated } \\
\text { position }\end{array}$ & Score & Protease & \multicolumn{1}{c}{ Peptide } \\
\hline 77 & 43 & Trypsin & K*IYPTIWWLFR \\
& 33 & Chymo & AKKK*IYPTIW \\
112 & 31 & Trypsin & K*QSEPFFK \\
& 25 & Chymo & TVADIRK*QSEPF \\
119 & 37 & Trypsin & KQSEPFF* \\
& 31 & Chymo & FK*ATPEEKL31 \\
& 31 & Chymo & K*ATPEEKL \\
201 & 56 & Trypsin & IIVEK*PFGR \\
& 26 & Chymo & NRIIVEK*PF \\
235 & 33 & Trypsin & IDHYLGK* \\
& 26 & Chymo & LGK*EMVQNL \\
390 & 46 & Trypsin & CGK*ALNER \\
396 & 26 & Chymo & NERK*AEVRLQF \\
416 & 24 & Trypsin & LQFHDVAGDIFHQQCK*R \\
& 27 & Chymo & HQQCK*RNEL \\
438 & 47 & Trypsin & K*PGMFFNPEESELDLTYGNR \\
459 & 28 & Chymo & K*NVKLPDAY \\
& 43 & Chymo & K*NVKLPDAYERL \\
462 & 22 & Trypsin & NVK*LPDAYER \\
527 & 78 & Trypsin & GPTEADELMK*R \\
& 61 & Trypsin & GPTEADELMK*R \\
538 & 50 & Trypsin & VGFQYEGTYK*WVNPHK \\
544 & 26 & Chymo & KWVNPHK*L \\
& & &
\end{tabular}

accessible (Fig. 4). The ability of aspirin to acetylate G6PD in vitro suggests that it is a non-enzymatic chemical reaction, consistent with its ability to acetylate numerous other proteins in-vitro (23).

\section{Discussion}

The housekeeping enzyme G6PD is the major regulatory enzyme in the pentose phosphate pathway, which catalyzes the conversion of glucose-6-phosphate to 6-phosphoglucono- $\delta$-lactone with a concomitant reduction of $\mathrm{NADP}^{+}$. NADPH thus generated is essential for the neutralization of oxidative stress within the cellular milieu. In addition, the pentose phosphate pathway generates ribose sugars, which are required for nucleic acid synthesis, and has a major role in rapidly dividing normal cells, as well as in cancer cell growth. Our previous study demonstrated that aspirin acetylates G6PD in HCT 116 cells, and this was associated with a decrease in its enzyme activity; however, the mechanism underlying this inhibition was not clearly identified (13). The aim of the present study was two-fold: i) To compare aspirin-mediated G6PD acetylation and enzyme activity between HCT 116 and HT-29 cells; and ii) identify the
Table II. Location of aspirin-acetylated lysine residues identified in the present study and the naturally acetylated lysines on G6PD (25).

\begin{tabular}{lcc}
\hline $\begin{array}{l}\text { Aspirin-acetylated } \\
\text { lysine sites in } \\
\text { isoform } a \text { (long) }\end{array}$ & $\begin{array}{c}\text { Corresponding lysine } \\
\text { sites in isoform } b \\
\text { (short) }\end{array}$ & $\begin{array}{c}\text { Naturally } \\
\text { acetylated } \\
\text { sites }\end{array}$ \\
\hline K77 & K47 & \\
K112 & K82 & K89 \\
K119 & K89 & K171 \\
K201 & K171 & \\
K235 & K205 & \\
K390 & K360 & \\
K396 & K366 & K386 \\
K416 & K386 & K403 \\
K438 & K408 & \\
K459 & K429 & K432 \\
K462 & K432 & K497 \\
K527 & K497 & \\
K538 & K508 & K514 \\
K544 & K514 & \\
\hline
\end{tabular}

Aspirin-mediated lysine acetylation targets included six of the seven naturally acetylated lysine residues. ${ }^{a} \mathrm{~K} 205$ was identified as important for catalysis $(21) .{ }^{b} \mathrm{~K} 403$ is a naturally acetylated site, which is not found to be acetylated following aspirin treatment.

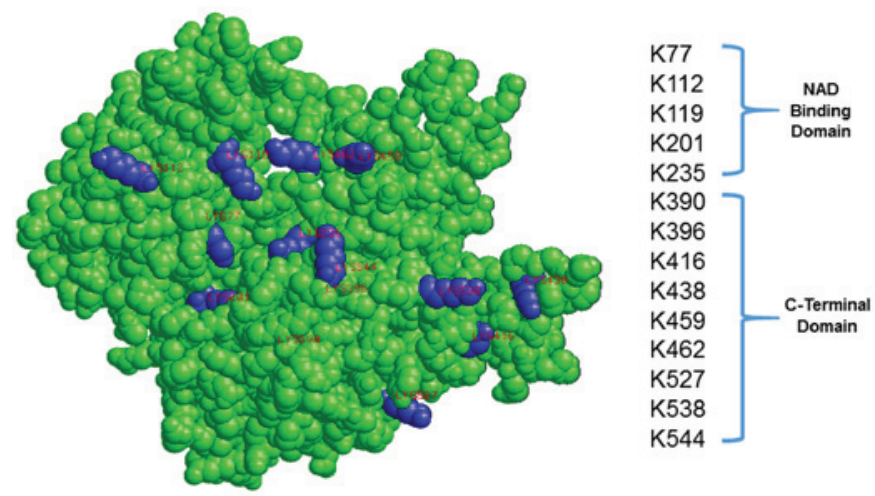

Figure 4. 3-Dimension space-filling model of recombinant glucose-6-phosphate dehydrogenase (G6PD; NP_000393), is shown. The location of aspirin-acetylated lysine residues are highlighted in blue (K77, K112, K119, K201, K235, K390, K396, K416, K438, K459, K462, K527, K538, K544).

aspirin-mediated acetylation targets in recombinant G6PD. The results demonstrated that exposure of HCT 116 cells to aspirin induced greater acetylation of G6PD compared with in HT-29 cells; accordingly, increased inhibition of G6PD activity was also observed in the HCT 116 cells. Although acetylated G6PD levels were lower in HT-29 cells, the G6PD protein levels (both isoform $a$ and $b$ ) were similar in both HCT 116 and HT-29 cells. The decreased levels of G6PD acetylation in HT-29 cells may be associated with a lower aspirin uptake, or more rapid hydrolysis of aspirin within the cells due to the action of esterases. Through MS analysis of 
in vitro acetylated recombinant G6PD (isoform $a$ ), a total of 14 lysine residues (K77, K112, K119, K201, K235, K390, K396, K416, K438, K459, K462, K527, K538, K544) were identified as targets of aspirin-mediated acetylation. These sites are dispersed throughout the length of the G6PD protein and are located in both the NAD-binding (aa 65-240) and the C-terminal domains (aa 242-536) (24). These results suggested that, in some cancer cell lines, aspirin may cause a direct inhibition of G6PD activity by modifying the protein via acetylation; and this may potentially contribute to reduced synthesis of ribose sugars and NADPH in cancer cells.

Within cells, G6PD exists as a mixture of monomer, dimer, tetramer and hexamer; but only the dimeric and tetrameric forms of the enzyme are catalytically active (25). The active enzyme exists in a dimer $\leftrightarrow$ tetramer equilibrium. It has previously been reported that G6PD isoform $b$ (short form) is naturally acetylated at seven lysine residues: K89, K171, K386, K403, K432, K497 and K514 (25,26). These modified lysines in isoform $b$ correspond to K119, K201, $\mathrm{K} 416$, K433, K462, K527 and K544 in G6PD isoform $a$ (long form). Among the naturally acetylated sites, and aspirin-acetylated sites, six of the seven sites appear to be common: K119, K201, K416, K462, K527 and K544. Notably, the acetylation of K235 by aspirin in G6PD isoform $a$ was observed in the present study. This corresponds to K205 in isoform $b$; this lysine residue has been demonstrated to be part of the active site of the enzyme, and is important in catalysis (21). K205 is located within the highly conserved peptide RIDHYLGK (residues 198-205, single letter amino acid code in isoform $b$ ) (27). Notably, in a previous study, it was reported that exposure of purified yeast G6PD to aspirin induced acetylation of a lysine within the G6PD peptide sequence IDHYLGK (aa 185-191), which resulted in inactivation of the enzyme activity (28). These previous reports $(21,28)$ along with the present findings, suggested that acetylation of this critical lysine (K205 in isoform $b$, and K235 in isoform a) important for catalysis may affect substrate binding (glucose-6-phosphate) and contribute to the aspirin-mediated inhibition of G6PD activity observed in HCT 116 cells. Alternatively, acetylation of other lysines may affect the affinity of NADP binding to G6PD, or simply may affect the dimer/tetramer formation required for keeping the enzyme in the active configuration. Molecular modeling of G6PD isoform $a$ suggested that the majority of aspirin-acetylated lysines are surface-exposed/solvent accessible (Fig. 4). Similar molecular modeling of isoform $b$ suggested that the corresponding amino acids (including K205 in the active site) are also surface-exposed (data not shown).

Wang et al (25) revealed that acetylation of K403 (in G6PD isoform $b$ ), mediated by cellular lysine acetyltransferases, negatively regulated enzyme activity. In addition, it was demonstrated that K403-acetylated G6PD is incapable of forming active dimers and displays a complete loss of activity. The K403 lysine in isoform $b$ corresponds to K433 in isoform $a$, and in the present MS analysis, this lysine was not identified as a target of aspirin. However, other lysine residues in close proximity, including K416 and K438, were acetylated following treatment with aspirin.

The present study demonstrated that aspirin acetylates G6PD, and this is associated with decreased enzyme activity, potentially due to modification of a lysine within the highly conserved peptide RIDHYLGK. These are important findings in the context of the known role of G6PD in cancer cell growth. G6PD has been reported to be overexpressed in numerous types of cancer, including breast, colon, endometrial, cervical, prostate and lung cancers $(18,19,29-32)$. Both NADPH and ribose-5-phosphates are essential products generated in the pentose phosphate pathway, which are important for cancer cell growth, through their contribution to nucleic acid biosynthesis and protection against oxidative stress. In addition, it has been reported that ectopic expression of G6PD in NIH3T3 cells significantly increased intracellular levels of NADPH and promoted anchorage-independent cell growth $(33,34)$. Due to its important role in cancer development, and increased expression in cancer, it is argued that G6PD may be a potential therapeutic target for cancer treatment (19). Based on the findings of the present study in HCT 116 cells, and the acetylation of K235 in isoform $a$ G6PD (equivalent to K205 in isoform $b$ ), it is likely that aspirin may inhibit G6PD activity in other cancer cell types; and therefore, may contribute to decreased cancer cell growth through reduced synthesis of ribose sugars and NADPH.

\section{Acknowledgements}

Support from the Translational Cancer Research Seed Grant, funded as the 2010 Research Initiative Center by the State of South Dakota, and from NIH (grant no. 5RO3CA133061-02) to GJB is gratefully acknowledged. The authors would also like to thank Mr. Raghavender Chivukula (Texas Tech University Health Science Center) for helpful discussions, and Dr. Fred Hagen (University of Rochester Medical Center, Rochester, NY) for carrying out the MS analysis.

\section{References}

1. Kaiser J: Will an aspirin a day keep cancer away? Science 337: 1471-1473, 2012.

2. Thun MJ, Jacobs EJ and Patrono C: The role of aspirin in cancer prevention. Nat Rev Clin Oncol 9: 259-267, 2012.

3. Chan AT, Arber N, Burn J, Chia WK, Elwood P, Hull MA, Logan RF, Rothwell PM, Schrör K and Baron JA: Aspirin in the chemoprevention of colorectal neoplasia: An overview. Cancer Prev Res (Phila) 5: 164-178, 2012.

4. Rothwell PM, Price JF, Fowkes FG, Zanchetti A, Roncaglioni MC, Tognoni G, Lee R, Belch JF, Wilson M, Mehta Z and Meade TW: Short-term effects of daily aspirin on cancer incidence, mortality, and non-vascular death: Analysis of the time course of risks and benefits in 51 randomised controlled trials. Lancet 379: 1602-1612, 2012.

5. Ferrández A, Piazuelo E and Castells A: Aspirin and the prevention of colorectal cancer. Best Pract Res Clin Gastroenterol 26: 185-195, 2012.

6. Alfonso L, Ai G, Spitale RC and Bhat GJ: Molecular targets of aspirin and cancer prevention. Br J Cancer 111: 61-67, 2014.

7. Dovizio M, Bruno A, Tacconelli S and Patrignani P: Mode of action of aspirin as a chemopreventive agent. Recent Results Cancer Res 191: 39-65, 2013.

8. Kopp E and Ghosh S: Inhibition of NF-kappa B by sodium salicylate and aspirin. Science 265: 956-959, 1994.

9. Hawley SA, Fullerton MD, Ross FA, Schertzer JD, Chevtzoff C, Walker KJ, Peggie MW, Zibrova D, Green KA, Mustard KJ, et al: The ancient drug salicylate directly activates AMP-activated protein kinase. Science 336: 918-922, 2012.

10. Choi HW, Tian M, Song F, Venereau E, Preti A, Park SW, Hamilton K, Swapna GV, Manohar M, Moreau M, et al: Aspirin's active metabolite salicylic acid targets high mobility group Box 1 to modulate inflammatory responses. Mol Med 21: 526-535, 2015. 
11. Dachineni R, Ai G, Kumar DR, Sadhu SS, Tummala H and Bhat GJ: Cyclin A2 and CDK2 as novel targets of aspirin and salicylic acid: A potential role in cancer prevention. Mol Cancer Res 14: 241-252, 2016.

12. Alfonso LF, Srivenugopal KS and Bhat GJ: Does aspirin acetylate multiple cellular proteins? (Review). Mol Med Rep 2: 533-537, 2009.

13. Marimuthu S, Chivukula RS, Alfonso LF, Moridani M, Hagen FK and Bhat GJ: Aspirin acetylates multiple cellular proteins in HCT-116 colon cancer cells: Identification of novel targets. Int J Oncol 39: 1273-1283, 2011.

14. Bateman LA, Zaro BW, Miller SM and Pratt MR: An alkyne-aspirin chemical reporter for the detection of aspirin-dependent protein modification in living cells. J Am Chem Soc 135: 14568-14573, 2013.

15. Wang J, Zhang CJ, Zhang J, He Y, Lee YM, Chen S, Lim TK, $\mathrm{Ng} \mathrm{S}$, Shen HM and Lin Q: Mapping sites of aspirin-induced acetylations in live cells by quantitative acid-cleavable activity-based protein profiling (QA-ABPP). Sci Rep 5: 7896, 2015.

16. Alfonso LF, Srivenugopal KS, Arumugam TV, Abbruscato TJ, Weidanz JA and Bhat GJ: Aspirin inhibits camptothecin-induced p21CIP1 levels and potentiates apoptosis in human breast cancer cells. Int J Oncol 34: 597-608, 2009.

17. Ai G, Dachineni R, Kumar DR, Marimuthu S, Alfonso LF and Bhat GJ: Aspirin acetylates wild type and mutant p53 in colon cancer cells: Identification of aspirin acetylated sites on recombinant p53. Tumor Biol Nov 23, 2015 (Epub ahead of print).

18. Stanton RC: Glucose-6-phosphate dehydrogenase, NADPH, and cell survival. IUBMB Life 64: 362-369, 2012.

19. Furuta E, Okuda H, Kobayashi A and Watabe K: Metabolic genes in cancer: Their roles in tumor progression and clinical implications. Biochim Biophys Acta 1805: 141-152, 2010.

20. Kanno H, Kondoh T and Yoshida A: 5' Structure and expression of human glucose-6-phosphate dehydrogenase mRNA. DNA Cell Biol 12: 209-215, 1993.

21. Bautista JM,MasonPJ and Luzzatto L:Humanglucose-6-phosphate dehydrogenase. Lysine 205 is dispensable for substrate binding but essential for catalysis. FEBS Lett 366: 61-64, 1995.

22. Karplus K: SAM-T08, HMM-based protein structure prediction. Nucleic Acids Res 37: W492-W497, 2009.

23. Pinckard RN, Hawkins D and Farr RS: In vitro acetylation of plasma proteins, enzymes and DNA by aspirin. Nature 219 : $68-69,1968$
24. Marchler-Bauer A, Derbyshire MK, Gonzales NR, Lu S, Chitsaz F, Geer LY, Geer RC, He J, Gwadz M, Hurwitz DI, et al: CDD: NCBI's conserved domain database. Nucleic Acids Res 43: Database Issue; D222-D226, 2015.

25. Wang YP, Zhou LS, Zhao YZ, Wang SW, Chen LL, Liu LX, Ling ZQ, Hu FJ, Sun YP, Zhang JY, et al: Regulation of G6PD acetylation by SIRT2 and KAT9 modulates NADPH homeostasis and cell survival during oxidative stress. EMBO J 33: 1304-1320, 2014.

26. Choudhary C, Kumar C, Gnad F, Nielsen ML, Rehman M, Walther TC, Olsen JV and Mann M: Lysine acetylation targets protein complexes and co-regulates major cellular functions. Science 325: 834-840, 2009.

27. Au SW, Gover S, Lam VM and Adams MJ: Human glucose-6-phosphate dehydrogenase: The crystal structure reveals a structural NADP (+) molecule and provides insights into enzyme deficiency. Structure 8: 293-303, 2000.

28. Jeffery J, Hobbs L and Jöernvall H: Glucose 6-phosphate dehydrogenase from Saccharomyces cerevisiae: Characterization of a reactive lysine residue labeled with acetylsalicylic acid. Biochemistry 24: 666-671, 1985.

29. Tsouko E, Khan AS, White MA, Han JJ, Shi Y, Merchant FA, Sharpe MA, Xin L and Frigo DE: Regulation of the pentose phosphate pathway by an androgen receptor-mTOR-mediated mechanism and its role in prostate cancer cell growth. Oncogenesis 3: e103, 2014.

30. Wang J, Yuan W, Chen Z, Wu S, Chen J, Ge J, Hou F and Chen Z: Overexpression of G6PD is associated with poor clinical outcome in gastric cancer. Tumour Biol 33: 95-101, 2012.

31. Du W, Jiang P, Mancuso A, Stonestrom A, Brewer MD, Minn AJ, Mak TW, Wu M and Yang X: TAp73 enhances the pentose phosphate pathway and supports cell proliferation. Nat Cell Biol 15: 991-1000, 2013.

32. Baba M, Yamamoto R, Iishi H, Tatsuta M and Wada A: Role of glucose-6-phosphate dehydrogenase on enhanced proliferation of pre-neoplastic and neoplastic cells in rat liver induced by N-nitrosomorpholine. Int J Cancer 43: 892-895, 1989.

33. Kuo W, Lin J and Tang TK: Human glucose-6-phosphate dehydrogenase (G6PD) gene transforms NIH 3T3 cells and induces tumors in nude mice. Int J Cancer 85: 857-864, 2000.

34. Kuo WY and Tang TK: Effects of G6PD overexpression in NIH3T3 cells treated with tert-butyl hydroperoxide or paraquat. Free Radic Biol Med 24: 1130-1138, 1998. 